\title{
$\int \ddot{\mathrm{OB}}$
}

Jahrbuch der Österreichischen Byzantinistik 


\title{
Herausgeber / Editors
}

\author{
Christian Gastgeber (ÖAW, ABF) \\ JoHANNES PREISER-KaPeller (ÖAW, ABF) \\ Claudia RapP (Universität Wien / ÖAW, ABF) \\ ELISABETH SCHIFFER (ÖAW, ABF) \\ Christian.Gastgeber@oeaw.ac.at \\ Johannes.Preiser-Kapeller@oeaw.ac.at \\ Claudia.Rapp@univie.ac.at \\ Elisabeth.Schiffer@oeaw.ac.at
}

Administrative Assistenz / Administrative Assistence

Petra Greger (Universität Wien)

INGRID WeICHSELbaUm (ÖAW, ABF)

Petra.Greger@univie.ac.at

Ingrid.Weichselbaum@oeaw.ac.at

ÖAW, ABF: Abteilung Byzanzforschung des Instituts für Mittelalterforschung der Österreichischen Akademie der Wissenschaften

A-1020 Wien, Hollandstraße 11-13, 4. Stock

Universität Wien: Institut für Byzantinistik und Neogräzistik der Universität Wien A-1010 Wien, Postgasse 7-9, 3. Stock

\section{Wissenschaftlicher Beirat / Advisory Board}

Wolfram Brandes (Frankfurt / M.), Leslie Brubaker (Birmingham),

Salvatore Cosentino (Bologna), Arne Effenberger (Berlin), Olivier Delouis (Paris), John Haldon (Princeton), Wolfram Hörandner (Wien), Sofia Kalopissi-Verti (Athen), Johannes Koder (Wien), Taxiarchis Kolias (Athen), Andreas E. Müller (Wien), Günter Prinzing (Mainz), Alexander Riehle (Princeton), Maciej Salamon (Krakau), Werner Seibt (Wien)

\section{Internet-Homepage}

http://www.austriaca.at/joeb_collection https://www.byzneo.univie.ac.at/publikationen/jahrbuch-der-oesterreichischen-byzantinistik/

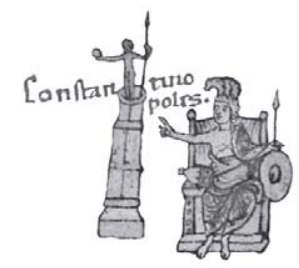




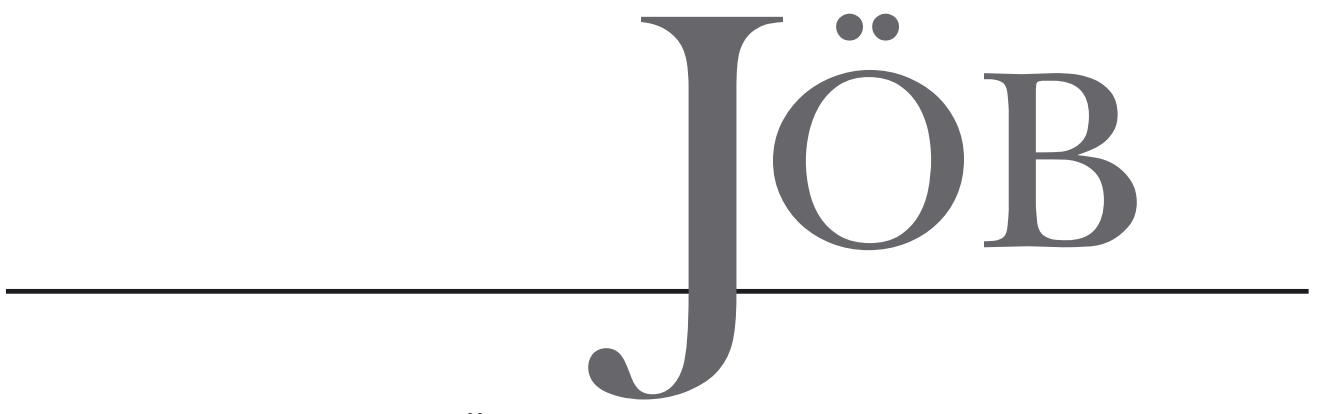

Jahrbuch der Österreichischen Byzantinistik

Band 68 / 2018 


\section{Jahrbuch der Österreichischen Byzantinistik}

Herausgegeben vom Institut für Mittelalterforschung, Abteilung Byzanzforschung der Österreichischen Akademie der Wissenschaften und vom Institut für Byzantinistik und Neogräzistik der Universität Wien

\section{Fortsetzung des Jahrbuches der Österreichischen Byzantinischen Gesellschaft}

Gedruckt mit Unterstützung der Historisch-Kulturwissenschaftlichen Fakultät der Universität Wien

\section{universität}

wien

Historisch-Kulturwissenschaftliche Fakultät

Bibliografische Information der Deutschen Nationalbibliothek

Die Deutsche Nationalbibliothek verzeichnet diese Publikation in der Deutschen Nationalbibliografie, detaillierte bibliografische Daten sind im Internet über http://dnb.d-nb.de abrufbar.

Lektorat: Nicola Wood (Englisch), Magdalena Delaunay (Französisch)

Diese Publikation wurde einem anonymen, internationalen Begutachtungsverfahren unterzogen.

Die verwendete Papiersorte in dieser Publikation ist DIN EN ISO 9706 zertifiziert und erfüllt die Voraussetzung für eine dauerhafte Archivierung von schriftlichem Kulturgut

Alle Rechte vorbehalten.

Copyright (C) Österreichische Akademie der Wissenschaften, Wien 2019

AU ISSN 0378-8660

ISBN 978-3-7001-8589-5

Satz: Crossdesign Werbeagentur GmbH (Graz), Christian Gastgeber (Wien)

Druck und Bindung: Prime Rate, Budapest

https://epub.oeaw.ac.at/joeb

https://verlag.oeaw.ac.at

Made in Europe 